\title{
Determination of the electronic density of states near buried interfaces: Application to Co/Cu multilayers
}

Nilsson, A.; Sthör, J.; Wiell, T.; Aldén, M.; Bennich, P.; Wassdahl, N.; Samant, M.G.; Parkin, S.S.P.; Mårtensson, N.; Nordgren, J.

Total number of authors:

12

Published in:

Physical Review B Condensed Matter

Link to article, DOI:

10.1103/PhysRevB.54.2917

Publication date:

1996

Document Version

Publisher's PDF, also known as Version of record

Link back to DTU Orbit

Citation (APA):

Nilsson, A., Sthör, J., Wiell, T., Aldén, M., Bennich, P., Wassdahl, N., Samant, M. G., Parkin, S. S. P.,

Mårtensson, N., Nordgren, J., Johansson, B., \& Skriver, H. L. (1996). Determination of the electronic density of states near buried interfaces: Application to Co/Cu multilayers. Physical Review B Condensed Matter, 54(4), 2917-2921. https://doi.org/10.1103/PhysRevB.54.2917

\section{General rights}

Copyright and moral rights for the publications made accessible in the public portal are retained by the authors and/or other copyright owners and it is a condition of accessing publications that users recognise and abide by the legal requirements associated with these rights.

- Users may download and print one copy of any publication from the public portal for the purpose of private study or research.

- You may not further distribute the material or use it for any profit-making activity or commercial gain

- You may freely distribute the URL identifying the publication in the public portal 


\title{
Determination of the electronic density of states near buried interfaces: Application to $\mathrm{Co} / \mathrm{Cu}$ multilayers
}

\author{
A. Nilsson \\ Department of Physics, Box 530, Uppsala University, S-751 21 Uppsala, Sweden \\ J. Stöhr \\ IBM Research Division, Almaden Research Center, 650 Harry Road, San Jose, CA 95120 \\ T. Wiell, M. Aldén, P. Bennich, N. Wassdahl \\ Department of Physics, Box 530, Uppsala University, S-751 21 Uppsala, Sweden \\ M. G. Samant and S. S. P. Parkin \\ IBM Research Division, Almaden Research Center, 650 Harry Road, San Jose, California 95120 \\ N. Mårtensson, J. Nordgren, and B. Johansson \\ Department of Physics, Box 530, Uppsala University, S-751 21 Uppsala, Sweden \\ H. L. Skriver \\ Physics Department, Technical University of Denmark, DK-2800 Lyngby, Denmark \\ (Received 14 September 1995; revised manuscript received 12 April 1996)
}

\begin{abstract}
High-resolution $L_{3}$ x-ray absorption and emission spectra of $\mathrm{Co}$ and $\mathrm{Cu}$ in $\mathrm{Co} / \mathrm{Cu}$ multilayers are shown to provide unique information on the occupied and unoccupied density of $d$ states near buried interfaces. The $d$ bands of both $\mathrm{Co}$ and $\mathrm{Cu}$ interfacial layers are shown to be considerably narrowed relative to the bulk metals, and for $\mathrm{Cu}$ interface layers the $d$ density of states is found to be enhanced near the Fermi level. The experimental results are confirmed by self-consistent electronic structure calculations. [S0163-1829(96)07628-X]
\end{abstract}

\section{INTRODUCTION}

The understanding of the electronic structure of interfaces is an important scientific and technological problem, since in many materials and structures interfaces play a key role in determining their physical properties, e.g., electronic transport and magnetism. In particular, it would be highly desirable to determine the electronic density of states near the Fermi level at real, buried interfaces. Unfortunately, the standard experimental techniques for such investigations, photoemission and inverse photoemission, are of limited value owing to their surface sensitivity and inability to discriminate between the density of states of different layers or elements. These problems are overcome by use of x-ray absorption (XAS) and x-ray emission (XES) spectroscopies, which are both element specific and can probe below the surface. The present paper illustrates the use of these techniques to determine the electronic density of states at interfaces in $\mathrm{Co} / \mathrm{Cu}$ multilayers, systems of great interest because of their unusual magnetotransport properties and oscillatory magnetic exchange coupling.

XAS and XES are complementary to photoemission and inverse photoemission spectroscopies in that they are local probes, owing to the local nature of the probed $2 p \leftrightarrow 3 d$ core-valence transitions. While the photoemission techniques may yield the $k$-dependent band structure, arising from the long-range periodic structure of the sample, XES and XAS provide a more local, chemical bonding, picture. Such a picture may prove valuable in addressing interfacial effects originating from short-range bonding and hybridization effects. XAS and XES yield information on the unoccupied and occupied $d$ states, respectively.

Here we present first results for the interfacial density of states in $\mathrm{Co} / \mathrm{Cu}$ multilayers. We find that the occupied $d$ bands of both $\mathrm{Co}$ and $\mathrm{Cu}$ interfacial layers in $\mathrm{Co} / \mathrm{Cu}$ multilayers are reduced in width relative to the pure metals. Also, the effect of hybridization of $\operatorname{Co~} d$ states with $\mathrm{Cu} s p$ states is directly observed. As a consequence, $\mathrm{Cu}$ interface atoms are found to exhibit an increased density of $d$ states across the Fermi level relative to bulk $\mathrm{Cu}$. These results are supported by a self-consistent $a b$ initio calculation of the electronic density of $d$ states. Our results explain the origin of the sizable magnetic moment on interfacial $\mathrm{Cu}$ atoms recently observed with $\mathrm{x}$-ray magnetic circular dichroism spectroscopy in $\mathrm{Co} / \mathrm{Cu}$ multilayers. ${ }^{6}$

\section{INITIAL- AND FINAL-STATE RULES}

In order to relate the local electronic structure to XES and $\mathrm{XAS}$ it is important to understand the influence of the core hole. Its effect has been summarized in the so-called "initial and final-state rules" for X-ray spectroscopies. ${ }^{2-4}$ These rules have been tested for metallic systems using model calculations for a homogenous electron gas. ${ }^{5}$ Figure 1 illustrates how X-ray spectra are related to the occupied and unoccupied quasiparticle $3 d$ density of states (DOS) corresponding to the removal and addition of an electron in the valence band, respectively. The quasiparticle DOS's are shown for a hypo- 

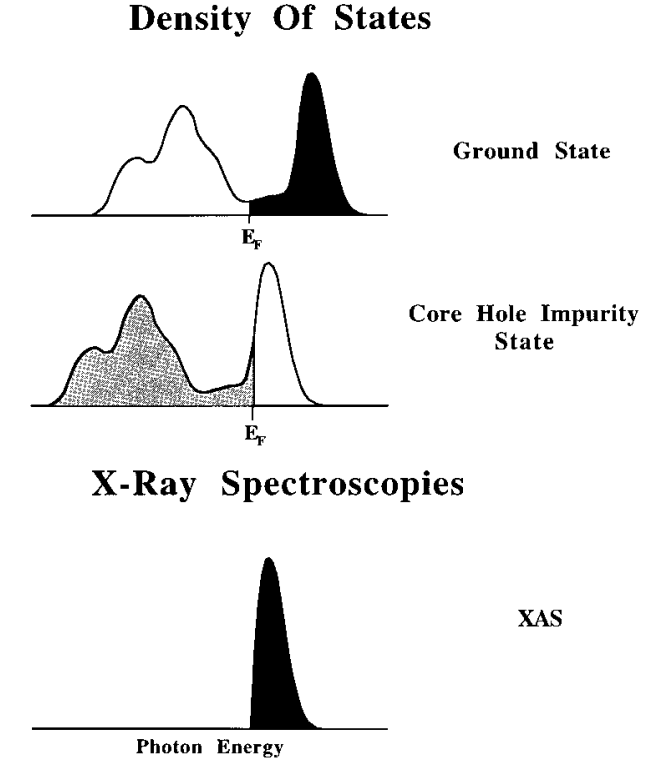

XAS

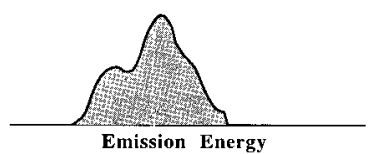

XES

FIG. 1. Schematic illustration of the $3 d$ density of states for a metal in the ground state and around an excited atom in a core hole impurity state and their relation to x-ray emission (XES) and absorption (XAS) spectra. Note that the filled black and gray areas are the same, respectively, according to the initial state rule.

thetical metal, both in the ground state and around a core hole impurity atom in the metal. For simplicity, the effect of the core hole on the local DOS is only shown as a movement of the position of the Fermi level by one electron per atom. However, in the real case the shape of the local DOS will be different for the core hole impurity atom. This can be understood by using the $Z+1$ approximation, which states that the core excited atom can be replaced by a $Z+1$ impurity atom. For Co metal, for example, the respective DOS's would correspond to $\mathrm{Co}$ metal and a $\mathrm{Ni}$ impurity atom in Co metal.

In a one-electron picture the XES spectrum reflects the occupied and the XAS spectrum the unoccupied local density of states. The position of the Fermi level in the spectrum, to which the valence electron binding energies are related, is given by the core-level photoemission binding energy. ${ }^{7}$ The final-state rule states that the spectral features reflect the eigenstates of the final-state Hamiltonian. For XAS a core hole is present in the final state and therefore the spectral line shape is mainly determined by the unoccupied DOS in the core hole impurity state. In XES, on the other hand, the core hole is filled by a valence electron leading to a final state related to the ground state. The final state in XES contains the quasiparticle valence hole similar to the final state in valence-band photoemission.

The initial-state rule states that the integrated intensity of a resonancelike feature in the spectrum, corresponding to a core $\leftrightarrow$ valence transition, is determinded by the total number of valence states in the initial state, i.e., prior to the x-ray transition. For XAS, the initial state corresponds to the ground state and the integrated x-ray intensity reflects the total number of empty valence states (holes) of symmetry determined by the dipole selection rule. For $L$-shell excitation in transition metals, for example, the XAS intensity corresponds to the number of $d$ holes (the transition intensity to $s$ holes is about a factor of 20 weaker) in the valence band, as indicated in Fig. 1. In XES, the initial state is influenced by the core hole and the integrated spectral intensity therefore reflects the total number of occupied states in the presence of the core hole.

In order to extract information relating to the ground-state electronic structure (without the core hole) the two x-ray spectroscopies complement each other. The spectral distribution in XES provides the DOS of the filled states and the integrated intensity in the XAS spectra provides the total number of valence holes.

For a more detailed description of the spectrum it is necessary to consider the dynamics of the excitation/ deexcitation process. The dynamic effects may alter the spectral shapes relative to the final state density of states. In a correct treatment one must consider the transition matrix element between the initial state and each final state. In that case all modifications of the wave functions that are caused by the electron excitation will contribute to the matrix element. From model calculations of simple metals, the dynamic effects tend to build up additional intensity close to threshold, which takes the form of a singularity at the Fermi level. ${ }^{5}$ Furthermore, in some transition-metal materials (oxides, etc.) the $L$ edge XAS spectra are determined to a large extent by $2 p-3 d$ multiplet effects. ${ }^{8}$ However, in the pure metals multiplet effects seem to be of less importance.

\section{EXPERIMENT}

All samples were grown by dc magnetron sputtering as for previous investigations. 1,6 "Bulk', samples of $\mathrm{Co}$ and $\mathrm{Cu}$ of 200 - $\AA$ thickness were grown on $\mathrm{Si}(100)$ using a $100-\AA \mathrm{Ru}$ buffer layer and a 20 - $\AA$ Ru capping layer to prevent oxidation. The $\mathrm{Co} / \mathrm{Cu}$ multilayer samples were of the structure $\mathrm{Si}(100) / \mathrm{Ru}(50 \AA) / 20 \times\{\mathrm{Co}(10 \AA) / \mathrm{Cu}(4 \AA)\} / \mathrm{Ru}(15 \AA)$ and $\mathrm{Si}(100) / \mathrm{Ru}(30 \AA) / 30 \times\{\mathrm{Co}(2.5 \AA) / \mathrm{Cu}(9.25 \AA)\} / \mathrm{Ru}(10 \AA)$. We shall refer to these samples as $\mathrm{Cu}(4) / \mathrm{Co}(10)$ and $\mathrm{Co}(2.5) /$ $\mathrm{Cu}(10)$. The experiments were carried out on the wiggler beam line 10-1 at the Stanford Synchrotron Radiation Laboratory (SSRL) equipped with a spherical grating monochromator (1000-1/mm grating). X-ray absorption measurements were carried out with a spectral resolution of $\sim 0.5 \mathrm{eV}$ by measuring the sample current (total electron yield). The $\mathrm{x}$-ray emission spectrometer consisted of an entrance slit, three spherical diffraction gratings, and a two-dimensional position-sensitive multichannel detector. ${ }^{9}$ It was oriented with its optical axis perpendicular to the incident $\mathrm{x}$ rays, in a vertically dispersive geometry. The incident linearly polarized $\mathrm{x}$ rays had an energy width of $\sim 5 \mathrm{eV}$ and at $50-\mathrm{mA}$ storage ring current the incident flux was about $1 \times 10^{12}$ photons/sec in the $800-1000-\mathrm{eV}$ range. The emission spectrometer was operated with a $1200-1 / \mathrm{mm}$ grating in second order with a spectral resolution of $\sim 0.5 \mathrm{eV}$ (entrance slit widths of $12 \mu \mathrm{m}$ for $\mathrm{Co}$ and $10 \mu \mathrm{m}$ for $\mathrm{Cu}$ ). X-ray emission spectra were recorded by use of threshold excitation, i.e., tuning the incident $\mathrm{x}$-ray energy to the $L_{3}$ absorption maxi- 


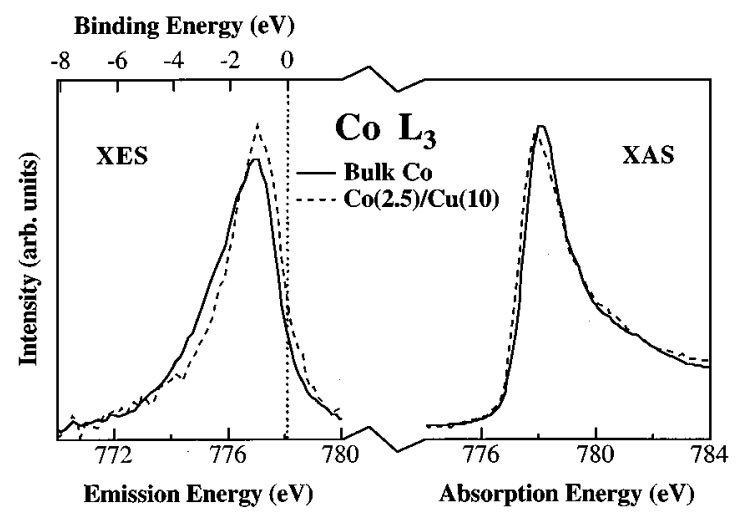

FIG. 2. Co $L_{3}$ x-ray emission (XES) and absorption (XAS) spectra of a $200-\AA$ Co film, denoted bulk Co, and the multilayer $\mathrm{Si}(100) / \mathrm{Ru}(30 \AA) / 30 \times\{\mathrm{Co}(2.5 \AA) / \mathrm{Cu}(9.25 \AA)\} / \mathrm{Ru}(10 \AA)$, denoted $\mathrm{Co}(2.5) / \mathrm{Cu}(10)$. The energy scales of the spectra were determined as discussed in the text. The Co $2 p_{3 / 2}$ XPS binding energy relative to the Fermi level was taken to be $778.1 \mathrm{eV}$.

mum, and with broad bandpass radiation ("white light"). In order to minimize self-absorption of the emitted $\mathrm{x}$ rays a $5^{\circ}$ grazing $\mathrm{x}$-ray incidence and near-normal $\mathrm{x}$-ray emission geometry was employed.

\section{RESULTS AND DISCUSSION}

Experimental XES spectra, obtained with threshold excitation, and electron yield XAS spectra for Co metal and a $\mathrm{Co}(2.5) / \mathrm{Cu}(10)$ multilayer are shown in Fig. 2. The absolute energy scale for the Co metal XES spectrum was established by placing the inflection point at the high-energy side of the $L_{3}$ emission resonance at the $2 p_{3 / 2}$ binding energy relative to the Fermi level $\left(E_{B}=778.1 \mathrm{eV}\right) .^{10}$ This establishes a binding energy scale relative to the Fermi level as shown on top, where the Fermi level cuts the $d$ band, as in X-ray photoemission spectroscopy (XPS). ${ }^{11}$ The energy alignment of the $\mathrm{Co}(2.5) / \mathrm{Cu}(10)$ spectrum relative to that of bulk Co was kept as measured and is precise to $0.1 \mathrm{eV}$. The XES intensities for bulk $\mathrm{Co}$ and $\mathrm{Co}(2.5) / \mathrm{Cu}(10)$ were scaled using the XAS results. For the XAS spectra the shown $L_{3}$ resonance intensities correspond to a per-atom basis and are therefore directly related to the number of unoccupied $d$ states. This was accomplished by normalizing the XAS spectra, recorded over a wide energy range, to the same edge jump across the $L_{3}$ and $L_{2}$ edges. ${ }^{6,12}$ The XAS results show that, within experimental accuracy, the number of empty $d$ states is the same for Co metal and Co in the multilayer. We have therefore scaled the two XES spectra to yield the same integrated intensities, reflecting an equal number of filled $d$ states.

Experimental results for bulk $\mathrm{Cu}$ and $\mathrm{Cu}(4) / \mathrm{Co}(10)$ are shown in Fig. 3. The absolute energy scales were obtained relative to the $2 p_{3 / 2}$ binding energy $E_{B}=932.4 \mathrm{eV}$ for $\mathrm{Cu}$ metal, ${ }^{10}$ following the same procedure as for Co. The XES scale was chosen so that the center of the $d$ band falls $3.0 \mathrm{eV}$ below the Fermi level, as determined by XPS, ${ }^{11}$ i.e., at 929.4 $\mathrm{eV}$. The relative energy scales of the $\mathrm{Cu}$ metal and $\mathrm{Cu}(4)$ / $\mathrm{Co}(10)$ spectra were kept as measured and, again, are precise

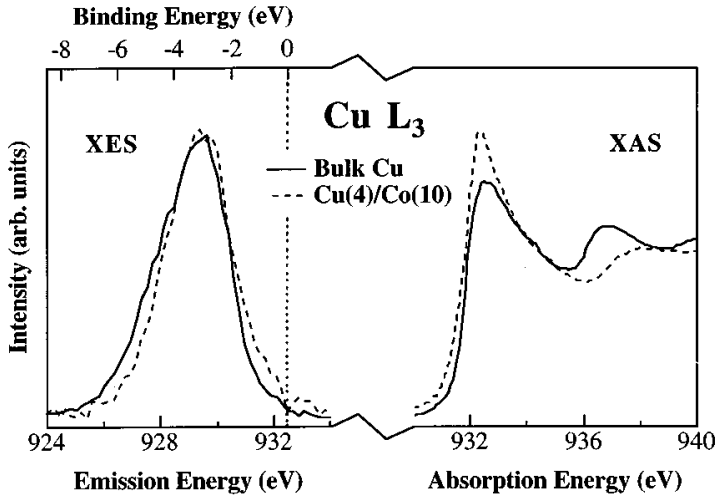

FIG. 3. $\mathrm{Cu} L_{3}$ X-ray emission (XE) and absorption (XA) spectra of a $200-\AA \mathrm{Cu}$ film, denoted bulk Cu , and the multilayer $\mathrm{Si}(100) /$ $\mathrm{Ru}(50 \AA) / 20 \times\{\mathrm{Co}(10 \AA) / \mathrm{Cu}(4 \AA)\} / \mathrm{Ru}(15 \AA)$, denoted $\mathrm{Cu}(4) /$ $\mathrm{Co}(10)$. The energy scales of the spectra were determined as discussed in the text. The $\mathrm{Cu} 2 p_{3 / 2}$ XPS binding energy relative to the Fermi level was taken to be $932.4 \mathrm{eV}$.

to $0.1 \mathrm{eV}$. The XES intensities were scaled as discussed above for $\mathrm{Co}$, considering the fact that the $d$ band is nearly $(\geqslant 95 \%$ ) filled in $\mathrm{Cu}$. Hence the pronounced change of the $\mathrm{Cu} L_{3}$ XAS resonance intensity between $\mathrm{Cu}$ metal and $\mathrm{Cu}$ in $\mathrm{Cu} / \mathrm{Co}$, while reflecting a sizable change in the number of empty $\mathrm{Cu} d$ states, still corresponds to a nearly equal number of filled $d$ states.

Since the experimental XES and XAS spectra overlap in energy the XES intensities may be reduced by selfabsorption on the high-energy side. We have calculated such effects for our experimental geometry and the various samples. The self-absorption effects at the $\mathrm{Cu} L_{3}$ edge were $5.3 \%$ versus $6.8 \%$ for $\mathrm{Cu}$ metal and $\mathrm{Cu}(4) / \mathrm{Co}(10)$, respectively, and at the Co $L_{3}$ edge $8 \%$ versus $7.8 \%$ for Co metal and $\mathrm{Co}(2.5) / \mathrm{Cu}(10)$. Hence in the comparison of the bulk and multilayer spectra in Figs. 2 and 3 the relative changes in self-absorption effects are negligible.

The XES spectra in Figs. 2 and 3 reveal that for both Co and $\mathrm{Cu}$ interfacial layers the $d$ bandwidths decrease relative to the bulk values and the line shapes become more symmetric. The effect is most pronounced for $\mathrm{Co}(2.5) / \mathrm{Cu}(10)$ in Fig. 2, where the Co $d$ bandwidth is about $0.5 \mathrm{eV}$ less than in bulk $\mathrm{Co}$. For $\mathrm{Cu}(4) / \mathrm{Co}(10)$ another interesting effect is observed in the $\mathrm{Cu}$ XES spectra, namely, an increase in the occupied density of states relative to bulk $\mathrm{Cu}$, in the region between the main $d$ band and the Fermi level. $\mathrm{Cu}$ XAS spectra reveal a similar effect for the integrated unoccupied density of $d$ states. Here the $L_{3}$ threshold resonance, whose intensity is a measure of the number of unoccupied $d$ states, is found the be markedly increased for $\mathrm{Cu}(4) / \mathrm{Co}(10)$ relative to bulk $\mathrm{Cu}$. In contrast, XAS results for Co interface atoms in $\mathrm{Co}(2.5) / \mathrm{Cu}(10)$ and for bulk Co show that the number of unoccupied $d$ states is nearly the same in both cases.

The experimental results are in good accord with theoretical densities of $d$ states, shown in Fig. 4, obtained by means of self-consistent $a b$ initio local spin-density theory, using a spin-polarized Green's function technique within the tightbinding linear muffin-tin orbitals method (LMTO). ${ }^{13}$ This 


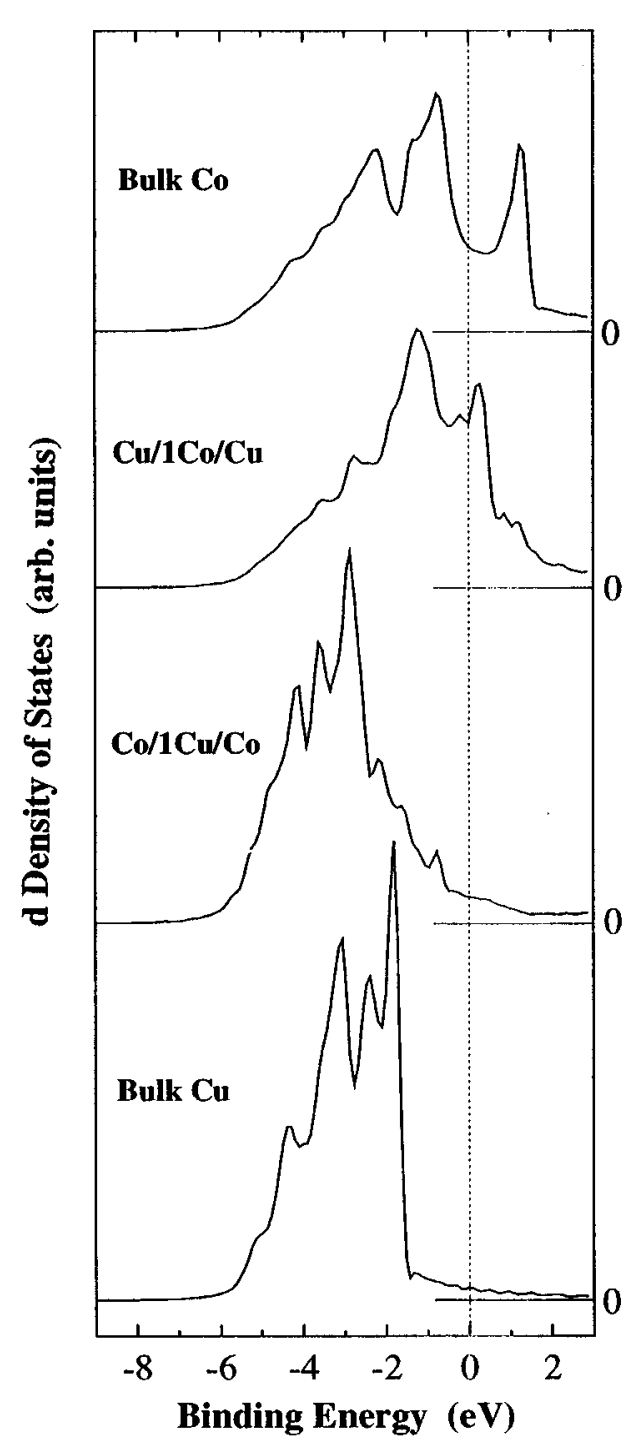

FIG. 4. Calculated density of $d$ states (sum of up and down spins) for ferromagnetic bulk hcp Co metal, a (111) monolayer of Co sandwiched between bulk fcc $\mathrm{Cu}$ (stacking sequence $A B C$ ), a (0001) monolayer of $\mathrm{Cu}$ sandwiched between bulk hcp Co (stacking sequence $A B A B$ ), and bulk fcc $\mathrm{Cu}$ metal.

method is inherently adapted to the interface-sandwich problem, since it takes proper account of the breakdown of translational symmetry that occurs perpendicular to the interface, and it has, for instance, previously been applied to magnetic multilayer calculations. ${ }^{14}$ The present formulation ${ }^{13}$ of the LMTO theory allows an unambigious projection of the state density and occupation numbers into site- and angularmomentum-labeled contributions. ${ }^{15}$ Calculations were carried out for ferromagnetic hcp $\mathrm{Co}$ and for fcc $\mathrm{Cu}$ in their perfect bulk structures, and for monolayers of $\mathrm{Co}(\mathrm{Cu})$ sandwiched between split $\mathrm{Cu}(\mathrm{Co})$ bulk crystals. The monolayers are constructed by embedding the single $\mathrm{Co}(\mathrm{Cu})$ layer between two semi-infinite bulk crystals of $\mathrm{Cu}(\mathrm{Co})$ and relaxing self-consistently the potentials for a chosen number of layers in the sandwich region through the Dyson equation. ${ }^{14}$ Here, it was found sufficient to include two layers on either side to

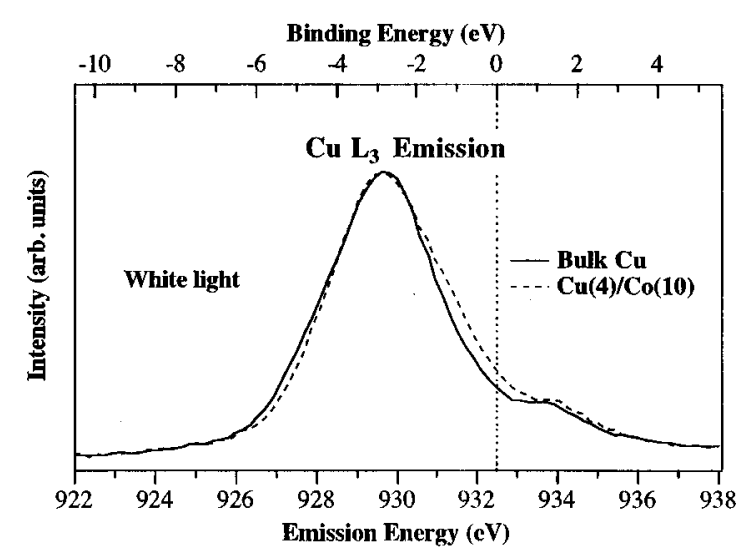

FIG. 5. $\mathrm{Cu} L_{3}$ XES spectra for bulk $\mathrm{Cu}$ (solid line) and $\mathrm{Cu}(4)$ / $\mathrm{Co}(10)$ (dashed line) excited with "white light," i.e., broad bandpass $x$ rays. Note sizable multielectron intensity above $932.4 \mathrm{eV}$, corresponding to the Fermi level position.

participate in the hybridization with the monolayer, yielding, in terms of sandwich thickness, complete convergence of the properties studied. We assumed a (111) fcc in-plane structure with an $A B C$ fcc-like stacking and the lattice constant for bulk $\mathrm{Cu}$ for $\infty \cdots 2 \mathrm{Cu} / 1 \mathrm{Co} / 2 \mathrm{Cu} \cdots \infty$, and the corresponding (0001) $A B A B$ hcp-like stacking and the lattice constant for bulk Co for $\infty \cdots 2 \mathrm{Co} / 1 \mathrm{Cu} / 2 \mathrm{Co} \cdots \infty$. The Green's functions for the sandwich structures were sampled on $90 k_{\|}$points in the irreducible part of the two-dimensional Brillioun zone. A possible relaxation of the layer spacings at the interface was neglected.

The theoretical results clearly exhibit the prominent changes observed experimentally for the thin interface layers. The $d$ bands for the sandwiched $\mathrm{Co}$ and $\mathrm{Cu}$ monolayers are narrowed and more symmetric relative to the bulk metals, and a sizable density of $d$ states extending through the Fermi level is found for the sandwiched $\mathrm{Cu}$ layer. All observed effects can be explained by a local hybridization picture. The narrowed interfacial density of $d$ states is similar to that observed previously by XPS for the surface layer on a clean metal surface, ${ }^{16}$ where it was attributed to a lowered coordination of surface relative to bulk atoms, resulting in a more atomiclike behavior. Our results show that a similar change in electronic density occurs at a buried interface. Although an interface atom does not necessarily have a different coordination number than a bulk atom the $d-d$ hybridization and therefore the $d$ bandwidth is expected to change owing to a difference in $d$ level binding energies of unlike atoms at an interface. The increased $d$ density of states near the Fermi level for interfacial $\mathrm{Cu}$ is attributed to hybridization of $\mathrm{Cu}$ states with $\mathrm{Co} d$ states, which exhibit their maximum density near the Fermi level. These results also explain the origin of the sizable magnetic $d$ moment on $\mathrm{Cu}$ interface atoms in $\mathrm{Co} / \mathrm{Cu}$ multilayers, recently observed by $\mathrm{x}$-ray dichroism. ${ }^{6}$

Finally, we show in Fig. 5 XES spectra for bulk $\mathrm{Cu}$ and $\mathrm{Cu}(4) / \mathrm{Co}(10)$ recorded with broad bandpass $\mathrm{x}$-ray excitation ("white light"). These spectra confirm the spectral changes observed with threshold excitation (Fig. 3), but the improved 
signal-to-noise ratio comes at the price of a sizable multielectron tail, ${ }^{17}$ which extends well above the $2 p_{3 / 2}$ binding energy $(932.4 \mathrm{eV})$ or, equivalently, the Fermi level position (zero binding energy). In particular, there is a strong CosterKronig satellite near $934 \mathrm{eV}$ resulting from excitation of the $2 p_{1 / 2}$ core state.

\section{SUMMARY}

Our studies show that XES studies following threshold excitation are uniquely capable of yielding the electronic density of states of buried layers and interfaces. In the future, the use of circularly polarized monochromatic $\mathrm{x}$ rays in the excitation process promises the determination of even the spin-resolved density of states ${ }^{18}$ at interfaces.

\section{ACKNOWLEDGMENTS}

The experimental part of this work was carried out in part at SSRL which is operated by the DOE, Division of Chemical Sciences. The computational aspects have emerged from fruitful collaborations with I.A. Abrikosov, S. Mirbt, and N.M. Rosengaard. The Uppsala workers would also like to acknowledge financial support by the Swedish Natural Science Research Council.
${ }^{1}$ S. S. P. Parkin, R. Bhadra, and K. P. Roche, Phys. Rev. Lett. 66, 2152 (1991).

${ }^{2}$ A. F. Starace, Phys. Rev. B 5, 1773 (1972); V. I. Grebennikov, Yu. A. Babanov, and O. B. Sokolov, Phys. Status Solidi B 79, 423 (1977); 80, 73 (1977).

${ }^{3}$ E. O. F. Zdansky, A. Nilsson, H. Tillborg, O. Björneholm, N. Märtensson, J. N. Andersen, and R. Nyholm, Phys. Rev. B 48, 2632 (1993); A. Nilsson and N. Martensson, Physica B 208/209, 19 (1995).

${ }^{4} \mathrm{~J}$. Stöhr and Y. Wu, in New Directions in Research with 3rd Generation Soft X-Ray Synchrotron Radiation Sources, Vol. 254, of NATO Advanced Studies Institute Series E: Applied Sciences, edited by A. S. Schlachter and F. J. Wuilleumier (Kluwer Academic, Dordrecht, 1994), p. 221.

${ }^{5}$ U. von Barth and G. Grossmann, Phys. Rev. B 25, 5150 (1982).

${ }^{6}$ M. G. Samant, J. Stöhr, S. S. P. Parkin, G. A. Held, B. D. Hermsmeier, F. Herman, M. van Schilfgaarde, L-C. Duda, D. C. Mancini, N. Wassdahl, and R. Nakajima, Phys. Rev. Lett. 72, 1112 (1994).

${ }^{7}$ A. Nilsson, O. Björneholm, E. O. F. Zdansky, H. Tillborg, N. Märtensson, J. N. Andersen, and R. Nyholm, Chem. Phys. Lett. 197, 12 (1992).

${ }^{8}$ F. M. F. de Groot, J. Electron Spectrosc. Relat. Phenom. 67, 529 (1994).

${ }^{9}$ J. Nordgren, G. Bray, S. Cramm, R. Nyholm, J.-E. Rubensson, and N. Wassdahl, Rev. Sci. Instrum. 60, 1690 (1989).
${ }^{10}$ J. Fuggle and N. Mårtensson, J. Electron Spectrosc. Relat. Phenom. 21, 275 (1980).

${ }^{11}$ S. Hüfner and G. K. Wertheim, Phys. Lett. 47A, 349 (1974).

${ }^{12}$ J. Stöhr, NEXAFS Spectroscopy, Springer Series in Surface Sciences Vol. 25 (Springer, Heidelberg, 1992).

${ }^{13}$ H.L. Skriver and N.M. Rosengaard, Phys. Rev. B 43, 9538 (1991); 46, 7157 (1992); M. Aldén, S. Mirbt, H.L. Skriver, N.M. Rosengaard, and B. Johansson 46, 6303 (1992). All important relativistic effects were included, except spin-orbit effects. The latter have been shown to have a negligible effect on the density of states by G. K. Wertheim, D. N. E. Buchanan, N. V. Smith, and M. M. Traum, Phys. Lett. 49A, 191 (1974).

${ }^{14}$ S. Mirbt, H.L. Skriver, M. Aldén, and B. Johansson, Solid State Commun. 88, 331 (1993).

${ }^{15}$ H.L. Skriver, The LMTO Method (Springer, Berlin, 1984).

${ }^{16}$ M. Mehta and C. S. Fadley, Phys. Rev. Lett. 39, 1569 (1977); Phys. Rev. B 20, 2280 (1979).

${ }^{17}$ N. Wassdahl, J.-E. Rubensson, G. Bray, P. Glans, P. Bleckert R. Nyholm, S. Cramm, N. Märtensson, and J. Nordgren, Phys. Rev. Lett. 64, 2807 (1990).

${ }^{18}$ P. Strange, P. J. Durham, and B. L. Gyorffy, Phys. Rev. Lett. 67, 3590 (1991); C. F. Hague, J.-M. Mariot, P. Strange, P. J. Durham, and B. L. Gyorffy, Phys. Rev. B 48, 3560 (1993); L.-C. Duda, J. Stöhr, D. C. Mancini, A. Nilsson, N. Wassdahl, J. Nordgren, and M. G. Samant, ibid. 50, 16758 (1994). 